\title{
Medical Management of Rumen Collapse in Cows
}

\author{
B. Gowri ${ }^{1}$, Juripriya Brahma ${ }^{1}$, C.S. Arunaman ${ }^{2}$ and D. Chandrasekaran ${ }^{2 *}$ \\ ${ }^{1}$ Department of Veterinary Clinical Medicine, Madras Veterinary College, Tamil Nadu Veterinary and Animal Sciences \\ University, Chennai, Tamil Nadu, INDIA \\ ${ }^{2}$ Department of Clinics, Madras Veterinary College, Tamil Nadu Veterinary and Animal Sciences University, Chennai, \\ Tamil Nadu, INDIA \\ *Corresponding author: D Chandrasekaran; E-mail: drchandrus73@gmail.com
}

Received: 11 Oct., 2019

Revised: 12 Nov., 2019

Accepted: 20 Nov., 2019

\begin{abstract}
Rumen collapse or rumen void is a syndrome usually observed in cattle affected with other primary diseases. It is a sequelae to other primary diseases due to total anorexia for several days. Recently purchased and calved animals brought into Large Animal Outpatient Medicine Unit, Madras Veterinary College Teaching Hospital, with the history of prolonged anorexia, mastitis and metritis were screened for rumen collapse. A total of 20 cases were included for the study. Animals were subjected to clinical, physical, rectal, haematological and biochemical examination. Anamnesis included were anorexia in all cases, high rise of temperature $15(75 \%)$ and high coloured urine $14(70 \%)$. An rumen collapsed in case of recently purchased animals were $12(60 \%)$. Clinical signs were anemia 18(90\%), enlarged lymph node 17(85\%), decreased rumen motility $17(85 \%)$, decreased or suspended rumination 16(80\%). Rectal examination revealed a small sized rumen (rumen collapsed). Rumen fluid examination revealedvery few live protozoa or complete absence of protozoal motility and increased in MBRT (Methylene blue reduction test) time. The haematological examination revealed reduced level of Hb, PCV and RBC level. Biochemical examination revealed reduced level of total protein and other parameters were remain in the normal range. The suggestive of the primary diseases involved were hypocalcaemia, theileriosis, anaplasmosis, mastitis, septic metritis, indigestion and babesiosis. The animals were firstly treated for primary diseases followed by rumen cud transplantation and oral administration of rumenotorics bolus.
\end{abstract}

Keywords: Cows, Rumen collapse, Rumen fluid examination

Rumen collapse is also called as "rumen void". The syndrome of rumen collapse is a sporadic condition usually observed in cattle affected with a primary severe inflammatory disease such as pneumonia, mastitis or metritis (Thomas and Simon, 2007). Rumen collapse was apparently caused by prolonged anorexia of several days associated with the primary inflammatory diseases. Cattle affected with rumen collapse had a rectangularshaped ping in the left upper quadrant of the abdomen. The simultaneous percussion and auscultation reveal a "ping" localized to the dorsal one-half to one -third of the left abdomen (Rebhun, 1987). This ping extends dorsally beyond the transverse processes of the lumbar vertebrae and includes the area of the paralumbar fossa and an area cranial to the paralumbar fossa covering up to four to five rib spaces. No fluid can be detected by ballottement in patients with rumen void. Rectal examination is necessary to confirm the problem and will reveal a collapsed dorsal sac of the rumen with no palpable rumen in the dorsal left quadrant and the left kidney pulled ventrally into the midabdomen.

If therapy for the primary disease is successful, the affected cow will begin to eat. The ventral extent of the ping will be located more dorsally each successive day during recovery as the rumen begins to fill and return to its normal position in the left upper quadrant. The prognosis is excellent if the

How to cite this article: Gowri, B., Brahma, J., Arunaman, C.S. and Chandrasekaran, D. (2019). Medical management of rumen collapse in cows. J. Anim. Res., 9(6): 907-911. 
primary disease is managed successfully because rumen collapse is a physiologic sign of prolonged anorexia rather than a pathologic GI disorder (Fubini and Divers, 2018).

\section{MATERIALS AND METHODS}

Twenty cattle affected with rumen collapse were selected from the Large Animal Outpatient Medicine Unit, Madras Veterinary College Teaching Hospital, with the history of prolonged anorexia and high coloured urine. Blood samples was collected from animals exhibiting clinical signs of pyrexia, anemia, enlarged lymph node, decreased rumen motility and decreased or suspended rumination. Blood samples were collected in EDTA anticoagulated vial for complete blood count and another 3 $\mathrm{ml}$ of blood sample were collected without anticoagulant in the vial for estimation of biochemical parameters. Peripheral blood smear was taken for examination of heamoprotozoan parasite. Complete blood count (CBC) was assessed with an automatic cell counter (Mindray BC Vet, 2800). Parameters assessed were: red blood cell count (RBC), hemoglobin ( $\mathrm{Hb}), \mathrm{PCV}, \mathrm{PLT}$ count, white blood cell count $(\mathrm{WBC})$ and $\mathrm{WBC}$ differential count including neutrophils, lymphocytes, monocytes, eosinophil. The serum concentration of alanine amino transferase (ALT), alkaline phosphatase (ALP), blood urea nitrogen (BUN), blood glucose, albumin, creatinine, total bilirubin and direct bilirubin were determined by automated serum biochemistry analyser (A-15 Biosystem) by using standard kits. Results obtained were expressed as means \pm standard deviation.

\section{Per rectal examination of the rumen}

Restrained the animal properly. Placement of the gloved and lubricated hand into the rectum by holding one hand in the shape of a cone and inserting the hand through the anus into the rectum. The fecal materials was removed manually, after that palpation of rumen was done.

\section{Rumen fluid analysis}

\section{Collection of rumen fluid}

A rumen fluid sample collected by rumenocentesis. Rumenocentesis or ruminalparacentesis is a technique involving percutaneous rumen puncture and aspiration of rumen fluid from the ventral ruminal sac (Petrovski, 2017). The landmark for rumenocentesis for insertion of the needle is the intersection of a horizontal line from the point of the patella and a vertical line $15-20 \mathrm{~cm}$ caudal to the last rib on the left side. Alternatively, rumenocentesis can be carried out over the left paramedian, just next to the umbilicus.

\section{Rumen fluid parameters Physical characters}

The rumen fluid was immediately examined after collection with regard to colour, odour and consistency. The colour and odour of rumen fluid were assessed by visual inspection and smell. The colourof rumen fluid is best assessedby transparent plastic or glass tube with a diameter $(<1.5 \mathrm{~cm})$. The sample should be closed in an airtight container for a minimum of five minutes and then opened to assess the odour (Petrovski, 2017).

Rumen fluid consistency is assessed by slowly turning a glass or plastic tube half-filled with rumen fluid $45-60^{\circ}$ left and right from an upright position. The assessment of the rumen fluid consistency should also assess for evidence of bubbles (Petrovski, 2017).

\section{Chemical characters}

The $\mathrm{pH}$ of rumen liquor sample examined using universal $\mathrm{pH}$ papers indicator immediately after collection of rumen fluid. Asmall quantity of rumen fluid was pouredupto the brim of the test tube after that a $\mathrm{pH}$ paper was inserted. The indicator paper was removed and the colour change was matched with standards of the indicator.

Methylene blue reduction time was estimated by transferring $20 \mathrm{ml}$ of rumen fluid in to a sterile glass test tube and mixing with $1 \mathrm{ml}$ of $0.03 \%$ methylene blue (Fubini and Ducharme, 2004). The mixed sample with test tube was allowed to stand at room temperature. The time in minutes needed for the colour of the mixture to change was recorded as methylene blue reduction time (Boodur et al., 2010).

\section{Microscopic examination of protozoa}

Protozoal activity was examined by placing one drop of fresh ruminal fluid on a microscope slide and a cover slip 
was place. It was examined under low power objective of magnifying microscope. Protozoan motility was graded in four categories: ++++ Good : $>10$ mobile protozoa per field; +++ fair: 6-9 mobile protozoa per field; ++ subnormal: 3-5 mobile protozoa per field; + very low: $<3$ mobile protozoa per field (Rosenberger et al., 1979).

\section{Gram staining}

Smear was made from rumen fluid and stained with Gram stained. Examined the stained smear under microscope.

\section{Treatment}

The animal was treated with primary diseases, followed by fluid therapy, osmotic ruminotoric (Magnesium Sulfate)@1g/kg body weight, rumen cud transfaunation and ruminotoric bolus treatment.

\section{RESULTS AND DISCUSSION}

A total of 20 cattle suffered from rumen collapse (Fig. 1) were diagnosed by history (Fig. 2), per rectal examination of rumen, clinical signs (Fig. 3) and infected with other primary disease (Fig. 4). The most prevalent clinical signs were anemia, enlarged lymph node, decreased rumen motility, decreased or suspended rumination and sunken of left paralumbar fossa (Fig. 3). The primary diseases were hypocalcaemia, theileriosis, anaplasmosis, mastitis, septic metritis, indigestion and babesiosis.

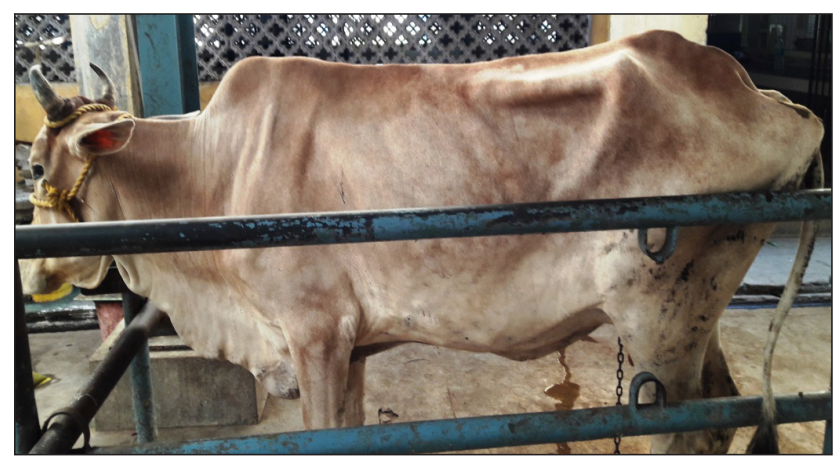

Fig. 1: Rumen collapse animal

The haematological examination reveals reduced level of $\mathrm{Hb}, \mathrm{PCV}$ and RBC level. Biochemical examination revealed reduced level of total protein and the other parameters were remain in the normal range.

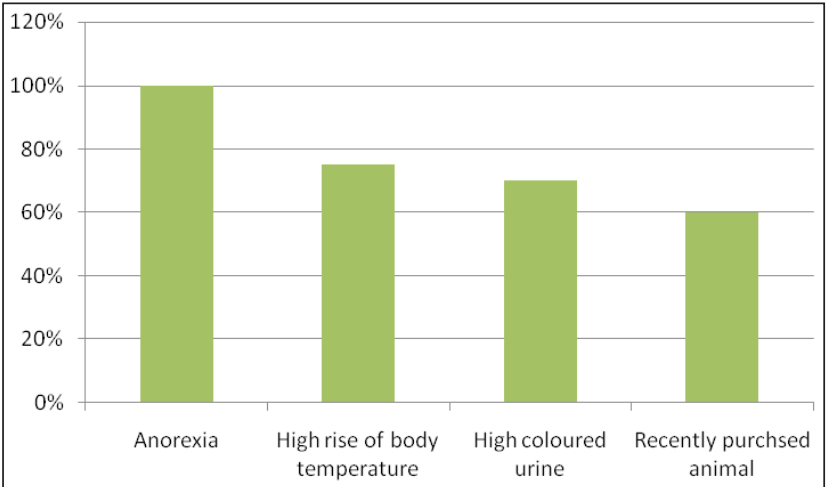

Fig. 2: History of rumen collapse case

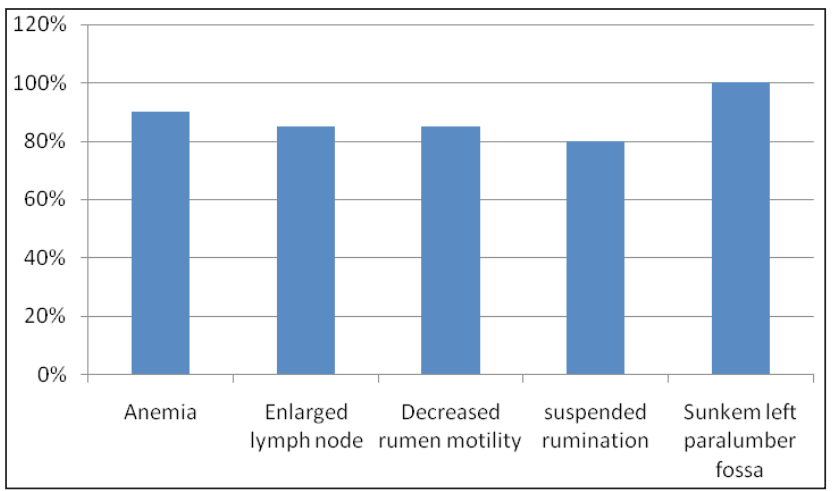

Fig. 3: Clinical signs of rumen collapse case

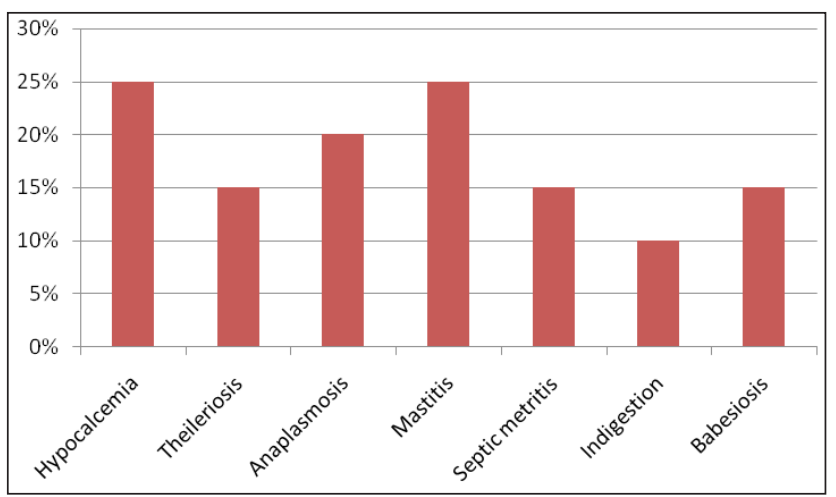

Fig. 4: Incidence of primary diseases

The haematological and biochemical results were expressed in Mean \pm SE (Table 1). Per-rectal examination, revealed a small sized rumen (rumen collapsed) Fig. 5. On auscultation and percussion of left side paralumbar fossa there was a pung sound. 
Table 1: Mean \pm SE value of haematological and biochemical parameters of rumen collapse cases

\begin{tabular}{cc}
\hline Parameters & Mean \pm SE \\
\hline Hb (mg/dl) & $6.56 \pm 0.0917$ \\
PCV $(\%)$ & $20.52 \pm 0.281$ \\
RBC (m/cmm) & $3.99 \pm 0.063$ \\
WBC (/cmm) & $7460 \pm 124.904$ \\
Platelets (/cmm) & $388300 \pm 4303.673$ \\
Neutrophil (\%) & $40.6 \pm 0.938$ \\
Lymphocyte (\%) & $2.51 \pm 0.957$ \\
Monocyte (\%) & $5.35 \pm 0.059$ \\
Glucose (mg/dl) & $63.65 \pm 0.62$ \\
Total protein (g/dl) & $6.26 \pm 0.033$ \\
BUN (mg/dl) & $19.66 \pm 0.196$ \\
Creatinine (mg/dl) & $1.01 \pm 0.013$ \\
Albumin (g/dl) & $3.1 \pm 0.012$ \\
Calcium (mg/d) & $9.14 \pm 0.09$ \\
AST (U/L) & $115.2 \pm 1.697$ \\
ALP (U/L) & $149.3 \pm 2.278$ \\
\hline
\end{tabular}

The colour of rumen fluid was normal yellowish brown colour. The odour was aromatic. The consistency of rumen fluid was watery and the finding was agreement with the finding of Petrovski, 2017. A very watery sample, with little particulate matter is indicative of starvation. The $\mathrm{pH}$ was ranged between 6-7 (Fig. 6A \& 6B).
Methyle Blue Reduction Test (MBRT) was shows prolonged time (15 minutes) for discolouration of rumen fluid colour. On microscopic examination of rumen fluid reveals very few live protozoa or complete absence of protozoal motility (Fig. 7), the finding was agreement with Petrovski, 2017. On Gram staining reveals predominant of gram negative bacteria.

The animal was treated with calcium borogluconate injection in case of hypocalcemia followed by Buparvaquone injection @ $2.5 \mathrm{mg} / \mathrm{kg}$ body weight in case of theileriosis, in anaplasmosis cases a course of oxytetracycline @ $10 \mathrm{mg} / \mathrm{kg}$ body weight were administered, specific antibiotic was administered on the basis of Antibiotic sensitivity test in case of mastitis, in case of septic metritis a broad spectrum antibiotic was administered and in babesiosis Diamenazine diaceturate (a) 3.5-7 mg/kg body weight was used.

In case of rumen collapsed fluid therapy, Ringers lactate (a) $15 \mathrm{ml} / \mathrm{kg}$ body weight, magnesium sulfate @ $1 \mathrm{~g} / \mathrm{kg}$ body weight, ruminotoric bolus i.e, Yea-Sacc bolus @, 2 boli/ day and rumen cud transfaunation was administered. After one week of treatment the animal was become normal health condition and the animal was taking food normally after post-examination of rumen fluid there was a normal protozoal population $(+++)$.

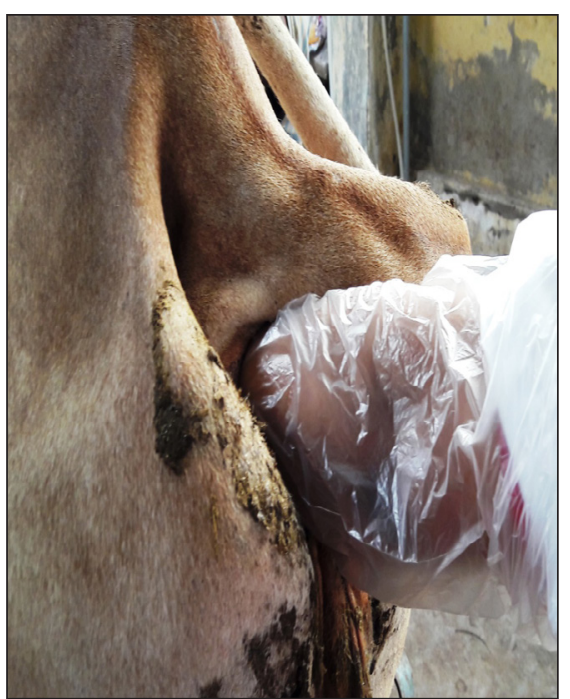

Fig. 5: Per rectal examination

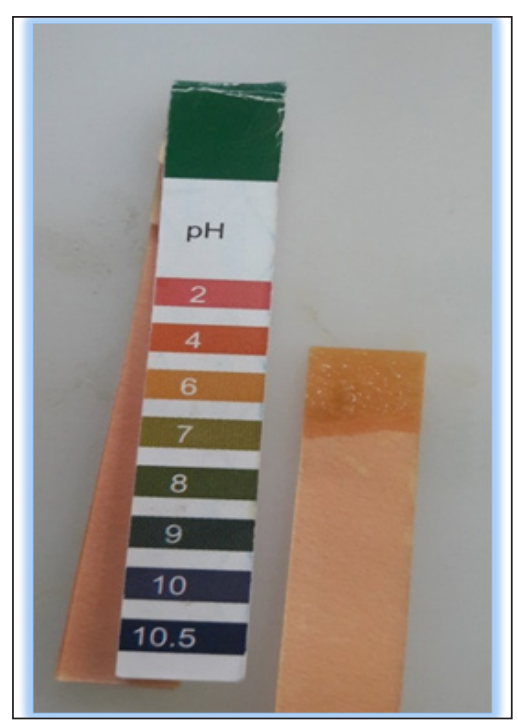

Fig. 6A: Rumen fluid showing $\mathrm{pH} 6$

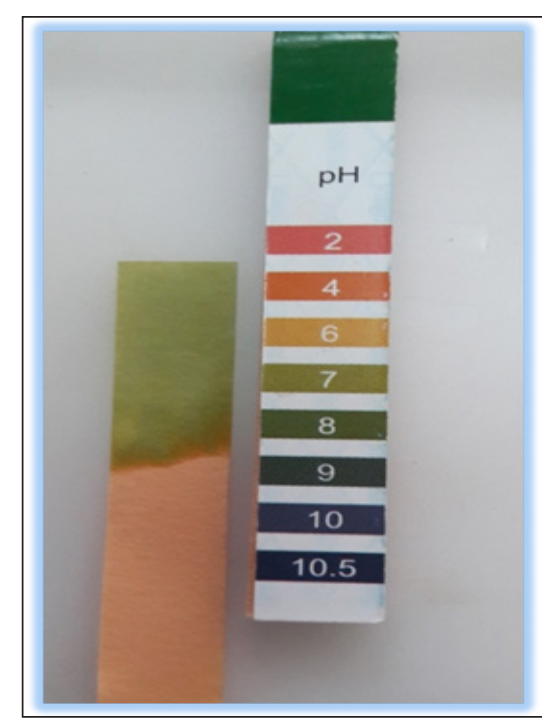

Fig. 6B: Rumen fluid showing $\mathrm{pH} 7$ 


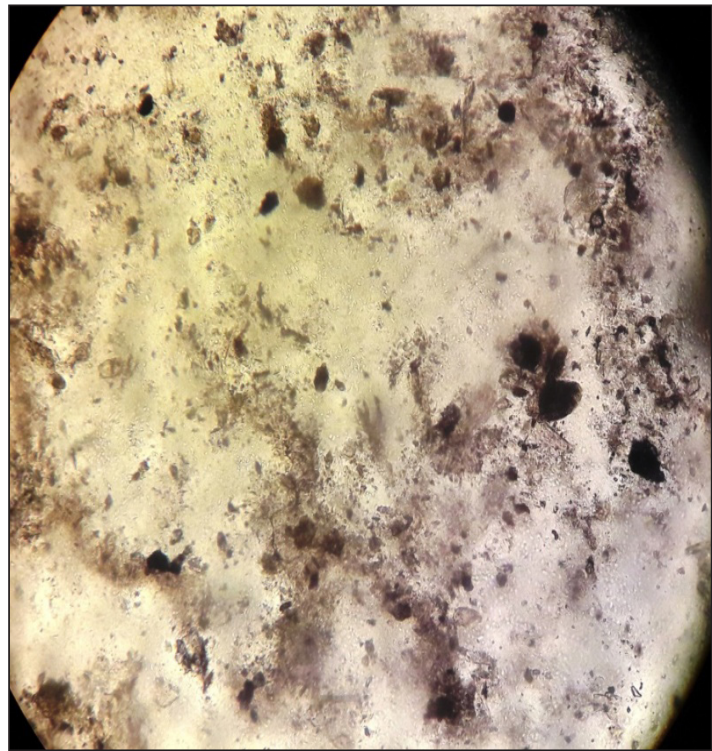

Fig. 7: Very few live protozoa in rumen collapse cases

\section{CONCLUSION}

Clinical signs of rumen collapse were anemia 18(90\%), enlarged lymph node $17(85 \%)$, decreased rumen motility $17(85 \%)$, decreased or suspended rumination $16(80 \%)$. Rectal examination revealed a small sized rumen (rumen collapsed). Rumen fluid examination revealed very few live protozoa or complete absence of protozoal motility and increased in MBRT (Methylene blue reduction test) time. The haemato biochemical examination revealed reduced level of $\mathrm{Hb}, \mathrm{PCV}$ and $\mathrm{RBC}$ and total protein levels and other parameters were within the normal range. The suggestive of the primary diseases involved were hypocalcaemia, theileriosis, anaplasmosis, mastitis, septic metritis, indigestion and babesiosis. The animals were firstly treated for primary diseases followed by rumen cud transplantation and oral administration of rumenotorics bolus.

\section{REFERENCES}

Boodur, P., Sivaprakash, B.V., Kasaralivar, V. and Rand D.D. 2010. Methylene blue reduction test in cattle affected with rumen impaction due to plastics. J. Ind. Poly Vet., 11(2): 184188

Fubini, S.L. and Ducharme, N.G. 2004. Surgery of ruminant fore-stomach compartment. In Farm Animal Surgery. S.L. Fubini and N.G. Ducharme (Eds), Saunders, Elsevier, pp. 161-240.

Rosenberger, G., Dirksen, H.D. Grunder, E., Grunert, D., Krauze, M. and Mack, R. 1979. Clinical Examination of Cattle. 2nd ed. Verky Paulparay, Berlin, Humburg, pp. 203-209.

Rebhun, W.C. 1987. Rumen collapse in cattle. Cornell Vet., 77(3): 244-250.

Thomas J.D. and Simon, F.P. 2007. Noninfectious Diseases of the Gastrointestinal Tract. Rebhun's Diseases of Dairy Cattle, $2^{\text {nd }}$ Edition, ch. 5, pp. 153-154. 
